\title{
The Initial 0.04 Second Polar Vector of the QRS Loop as an Aid in the Detection of a Myocardial Infarction
}

\author{
Dino A. Belletti, M. D.* and Alan F. Lyon, M. D.**
}

\section{SUMMARY}

Thirty-one normal subjects and 16 subjects with documented myocardial infarction were studied. The Frank system of vectorcardiography was used. The polar vector and 0.04 polar vector were calculated in all. The 0.04 polar vector was abnormally directed in all cases of documented infarction, including 2 subjects with a documented infarction but a "suggestive" electrocardiogram. The results of this preliminary study suggest that this method of vectorcardiographic analysis may be of a clinical value in those cases with a previous myocardial infarction in which the present electrocardiogram is suggestive but not diagnostic of an infarction. The results also demonstrate the greater diagnostic value of the 0.04 polar vector over the polar vector in detecting myocardial infarction.

\section{Additional Indexing Words:}

Vectorcardiogram Coronary artery disease Coplanarity of the QRS loop

\footnotetext{
CORRECTED or orthogonal lead sytems, displayed either as the scalar X, $\mathrm{I} \mathrm{Y}$ and $\mathrm{Z}$ leads or as vectorcardiographic loops, have had increasing clinical use. While these rather accurate displays of the single dipole information of the surface electrocardiogram have proved most useful in teaching, the additions they have made to the diagnostic accuracy of the standard 12 lead electrocardiogram have been limited. The information contained in loop display has proved too complex for the easy establishment of minimal diagnostic criteria ; computer analysis of scalar orthogonal leads, while potentially more useful, has not yet produced criteria allowing complete separation of normal tracings from those reflecting myocardial infarction.

From the Medical Service of the Veterans Administration Hospital, 130 West Kingsbridge Ruad, Bronx, New York 10468.

* Assistant Chief, Gardiology Section, Veterans Administration Hospital, Bronx, New York 10468.

** Former Chief, Cardiology Section, Veterans Administration Hospital, Bronx, New York 10468. Presently Chief of Cardiology, Brookdale Hospital Genter, Linden Boulevard at Brookdale Plaza, Brooklyn, New York 11212.

Received for publication December 10, 1968.
} 
For several years, the polar vector, the vector perpendicular to the mean plane of the spatial loop, has been studied. ${ }^{11}$ It has been demonstrated that overlap between normal and abnormal is considerable, and that the clinical applications of the study of this interesting quantity are likely to limited.2)

The study is a preliminary report of the use of a polar vector constructed to only the initial $0.04 \mathrm{sec}$. of the QRS loop in the separation of myocardial infarction from normal.

This report also demonstrates the sensitivity of the 0.04 polar vector in detecting myocardial infarction in subjects where the mean polar or polar vector is considered normal and the 12 lead electrocardiogram is not diagnostic.

\section{Materials and Methods}

A total of 47 subjects were studied. Ages ranged from 27 to 75 . Thirty-one were considered to have no clinical evidence of heart disease by history, chest X-ray and electrocardiograms. Sixteen subjects had a clinical story of an infarction with confirmation by electrocardiogram and enzyme elevation at some time in the past. Two of these at the time of study had only suggestive electrocardiographic evidence of infarction - in one a non-diagnostic $Q$ wave in $\mathrm{aV}_{\mathrm{F}}$ and in the other poor $\mathrm{R}$ wave progression over the precordial leads. One with a prior anteroseptal infarction had a completely normal electrocardiogram at the time of study.

The Frank orthogonal lead system was used with the subjects in the supine position and the chest electrodes placed in the fourth intercostal space as recommended. ${ }^{31,4)}$ The Electronics for Medicine Multichannel recorder with attached timing circuit was used to record and photograph the loops. The timing circuit permitted exclusion of $P$ and $T$ loops when desired. Standardization of the loops on the oscilloscope screen was performed for each subject. One millivolt was made to produce a $5 \mathrm{~cm}$. deflection. Photographs of the loops in the frontal transverse and left sagittal plane were obtained. For better delineation of early forces, a fourfold amplification of the isolated QRS loop was photographed. The polar vector was calculated by manual measurement of the area of the QRS loop in each plane-frontal, transverse and left sagittal. The area was calculated by the use of either a planimeter or by transferring the loop to graph paper and counting the squares encompassed.

If a plane has figure of eight inscription (both clockwise and counter clockwise), the areas were subtracted and net are calculated. If the major component was clockwise then the area was considered negative; if the area was counter clockwise it was considered positive. The coordinates of the polar vector, latitude (elevation) or angle $\psi$ (psi) and the longitude (Azimuth); or angle $\theta$ (theta), were calculated by the application of the following formulas $(4,5)$

$$
\phi=\tan ^{-1} \sqrt{\frac{\mathrm{X}^{2}+\mathrm{Z}^{2}}{\mathrm{Y}}} \quad \theta=\tan ^{-1} \mathrm{X}
$$

The $\mathrm{Z}$ coordinate of the polar vector was calculated by measurement of the frontal $(\mathrm{XY})$ area, which was considered negative if inscription was clockwise and 
positive if inscription was counter clockwise. The X coordinate of the polar vector was calculated from the sagittal plane. It was considered positive if the net area was counter clockwise and negative if clockwise. The $\mathrm{Y}$ coordinate of the polar vector was calculated from the transverse plane area and was considered positive if inscription counter clockwise and negative if clockwise. The 0.04 polar vector was determined in a similar fashion, but instead of calculating the total area of the loop in its three planes, only the 0.04 area (that area cnclosed by the perimenter of the loop and a striaght line from the $\mathrm{E}$ point to the 0.04 dot on the loop) was used.

\section{Results}

The distribution of the 0.04 polar vector of the 31 normal subjects was $\theta=35^{\circ}$ to $145^{\circ}$ and $\phi$ from $12^{\circ}$ to $67^{\circ}$.

The distribution of the 0.04 polar vector in the infarction group was $\theta=14^{\circ}$ to $75^{\circ}, 148^{\circ}$ to $178^{\circ}$ and $\phi=15^{\circ}$ to $143^{\circ}$.

The distribution of the mean polar vector of the normal subjects was $\theta=21^{\circ}$ to $156^{\circ}$ and $\phi$ from $20^{\circ}$ to $66^{\circ}$.

The distribution of the mean polar vector in the infarction group was $\theta=1$ to 179 and $\phi$ from $14^{\circ}$ to $135^{\circ}$.

Fig. 3 illustrates the distribution of the coordinates 0.04 polar vectors in

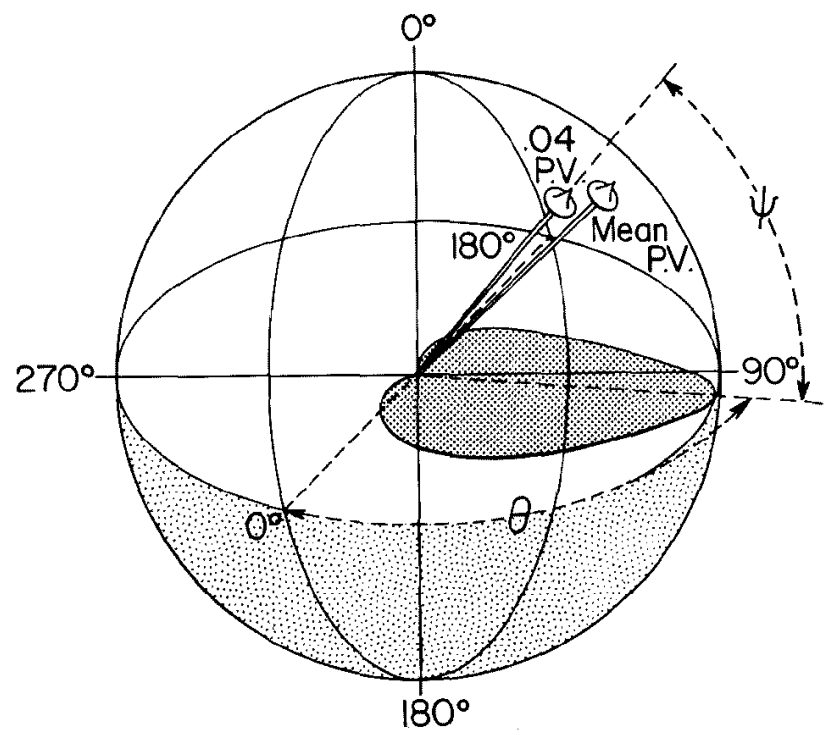

\section{NORMAL: \\ Spatial orientation of the .04 and Mean Polar Vector.}

Fig. 1. Note the flatness of a normal QRS loop. The spatial angle between the 0.04 polar and mean polar vector in the normal QRS loop is minimal. 
the normal and post infarction subjects and the distribution of the mean polar vector in normal and the post infarction group. The 0.04 polar vector in normals is represented by $\mathrm{X}$. The coordinates of the 0.04 polar vector of the infarct group is illustrated by the solid circle, and the mean polar vector of the normal group as a circle.

The separation of the normal and abnormal 0.04 polar vector is apparent. There is one determination in the infarction group (the subject with the normal electrocardiogram), whose coordinates are $\theta=146^{\circ}$ and $\psi=15^{\circ}$, close to the normal group.

The mean polar vectors of the abnormal group show a wide scatter and in 2 instances, their positions are well within the normal distribution.

The 2 subjects with the non-diagnostic electrocardiogram, the poor $R$ wave progression in one and a questionable $0.04 \mathrm{Q}$ wave in $\mathrm{aV}_{\mathrm{F}}$ in the other, with previous documented myocardial infarction, had a 0.04 polar vector directed distinctly away from the 0.04 polar vector of normal subjects.

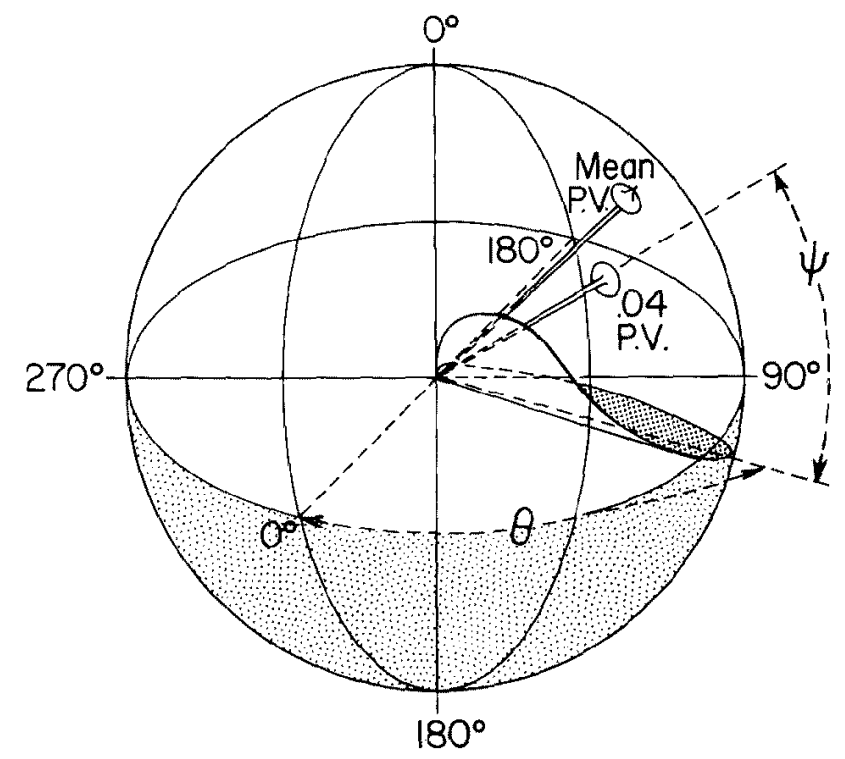

FOLLOWING INFARCTION:

Coplanarity of QRS loop is lost.

Mark change in spatial orientation of

.04 Polar Vector.

Minimal change of Mean Polar Vector.

Fig. 2. The spatial angle between the 0.04 polar and mean polar vector is increased. This increase is due mainly to a change in the orientation of the 0.04 polar vector in the infarction group. 


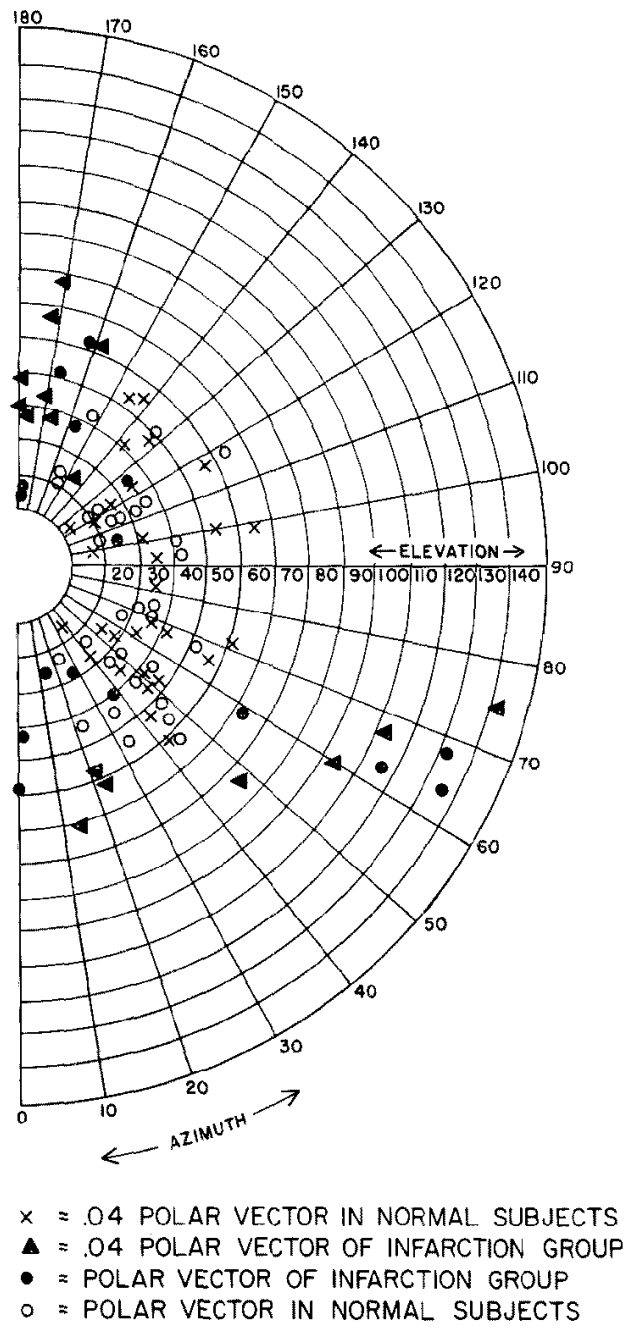

Fig. 3. The spatial orientation of the 0.04 and polar vector plotted on polar coordinates of both the normal and infarction group.

\section{Discussion}

A striking characteristic of the electrical representation of ventricular depolarization is that it can be represented by a vector loop with considerable coplanar symmetry. Normal spatial loops are thus nearly flat. We have observed similar flatness in abnormal vector loops from cases of hypertrophy or left bundle branch block. Inspection of spatial loops from cases with prior myocardial infarction, however, reveals that coplanar symmetry is lost, and that the initial position of the QRS is tilted in a different plane from that of the remainder of the QRS (Fig. 2). 
The polar vector, as the perpendicular to these planar surfaces, provides a useful way to study this change from normal. The polar vector represents a moment and thus includes both magnitude and direction. For the purposes of this study only direction was utilized in the analysis.

The separation of normal from infarction 0.04 polar vector can be accomplished in two ways-by comparing the orientation in the 2 groups directly or by comparing the spatial angles between the 0.04 polar vectors and the mean polar vectors in each group. The former approach was used in this preliminary report.

Fig. 3 displays the orientation of the 0.04 polar vector in the normal and infarction group as well as the mean polar vector in these 2 groups. These results indicate a nearly complete separation of the infarction group from the normal group when the 0.04 polar vector is utilized. The separation is considerably more complete than when the mean polar or polar vectors are compared.

In the 2 cases of previous myocardial infarction where the present 12 lead electrocardiogram is interpreted as suggestive of an infarction, the 0.04 polar vector has a direction distinctly separate from that determined for the normal group. The coordinates of the mean or polar vector in these 2 cases is within the normal range. More cases, however, are required with documented infarction without diagnostic electrocardiographic findings in order to fully evaluate the sensitivity of the orientation of the 0.04 polar vector as compared to the 12 lead electrocardiogram in such cases. In these 2 cases, the 0.04 polar vector is apparently superior, not only to the polar vector but also to the electrocardiogram.

A comparison of the accuracy of this criterion with that of deflection criteria in the scalar $\mathrm{X}, \mathrm{Y}$ and $\mathrm{Z}$ leads analyzed by comparison with a memory core of known infarcts and known normals in a digital computer is not available to us or indeed to most physicians.

These preliminary results would appear to warrant further study of the 0.04 QRS polar vector as a new and more precise tool for the diagnosis of myocardial infarction.

\section{REFERENCES}

1. Burger, H. C. and Vaane, J. P.: Am. Heart J. $56: 29,1958$.

2. Pipberger, H. V. and Carter, T. N.: Circulation 25: 538, 1962.

3. Frank, E.: Circulation 13: 737, 1956.

4. Langner, P. H., Jr., Okada, R. H., Moore, S. R., and Fies, H. L.: Circulation 17: 46, 1958.

5. Okamoto, N. and Simonson, E. : Am. J. Cardiol. 18 : 682, 1962. 